\title{
Theory of magnetic dissipation imaging
}

\author{
Y. Liu, B. Ellman, and P. Grütter ${ }^{\mathrm{a})}$ \\ Department of Physics, Centre for the Physics of Materials, McGill University, \\ Montréal, Québec H3A 2T8, Canada
}

(Received 7 February 1997; accepted for publication 3 July 1997)

\begin{abstract}
A model is presented for magnetic dissipation imaging and magnetic force gradient imaging obtained with a vibrating ferromagnetic tip and a ferromagnetic thin film sample. Results of calculations are compared to recent experiments and show good agreement using known bulk values for the magnetic parameters of tip and sample. We suggest that oscillations of domain wall width result in magnetoelastic emission of phonons. These phonons carry energy from the tip, leading to image contrast at domain walls. We also discuss the energy dissipation resulting from eddy current losses in the tip and sample. (C) 1997 American Institute of Physics. [S0003-6951(97)01936-0]
\end{abstract}

Magnetic force microscopy (MFM) has been used to measure local magnetic dissipation in magnetic samples for the first time by Grütter et al. ${ }^{1}$ An oscillating magnetic tip creates a highly localized alternating magnetic field when close to the sample. Magnetic energy dissipation in the sample leads to damping of the cantilever oscillation. The frequency, phase and amplitude of the cantilever oscillation are measured with a dedicated phase-lock loop. ${ }^{2}$ By controlling the phase and keeping the amplitude of the cantilever oscillation constant, the change in driving signal amplitude is directly proportional to dissipation. MFM and dissipation images can be acquired simultaneously. Several different samples (4 $\mathrm{nm}$ sputtered Co film on Si, $20 \mu \mathrm{m}$ Permalloy squares, sputtered $\mathrm{Co} / \mathrm{Ni}$ multilayers, Terfenol films and magnetic recording media) have been studied with this technique and resolutions better than $100 \mathrm{~nm}$ have been achieved. Strong correlations between the simultaneously measured domain structure and the dissipation images are observed.

Dissipation measured by this technique is equivalent to a measurement of the spatially resolved energy loss of a minor hysteresis loop. In this letter, we investigate the mechanism for energy dissipation and magnetic interaction between a ferromagnetic tip and a magnetic sample. We find that the major features of the dissipation images can be explained by magnetoelastic losses. Furthermore, by using the bulk values for the magnetic parameters characterizing the tip and the samples, the calculations based on this model are quantitatively in agreement with the experiments to within a factor of 2 .

The main features of the energy dissipation and the force gradient acting on the tip as it goes across a sample can be illustrated with the simple model sketched in Fig. 1(a). In this example, we consider uniaxial anisotropy and assume that the spins in the domain walls gradually rotate their direction from one domain to another with a constant angle between adjacent spins. The crucial part of this model is the external field dependence of the domain wall width. When neglecting the demagnetization energy, the domain wall energy per unit area contains exchange energy $\gamma_{e x}$ $=\left(J S^{2} \pi^{2} / a\right) \cdot(1 / w)$, anisotropy energy $\gamma_{a n}=\left(K_{1} / 2\right) \cdot w$

${ }^{\text {a)} E l e c t r o n i c ~ m a i l: ~ g r u t t e r @ p h y s i c s . m c g i l l . c a ~}$ and magnetostatic energy $\gamma_{s t a}= \pm\left(2 \mu_{0} M_{s} H_{y} / \pi\right) \cdot w$. Here $J$ is the exchange constant between adjacent spins of angular momentum $\hbar S, K_{1}$ and $a$ are the anisotropy constant and unit cell dimension of the sample, respectively, $w$ is the domain wall width, $M_{s}$ is the saturation magnetization of the sample, and $H_{y}$ is the in-plane external field which, for a vibrating magnetic tip, can be written as $H_{y}=H_{y 0}$ $+H_{y 1} \cos (2 \pi f t)$ with $f$ the resonant frequency of the cantilever; the "+" sign in $\gamma_{\text {sta }}$ is for wall B and the "-" sign
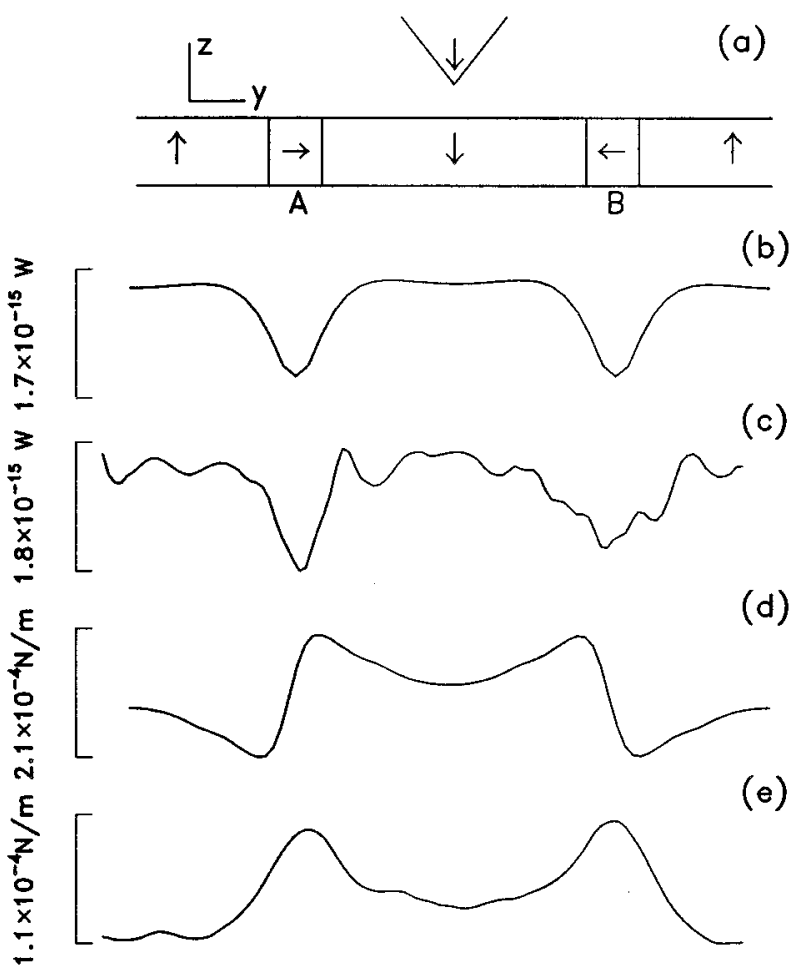

FIG. 1. Variation in magnetic dissipation and magnetic force gradient images from dissipation force microscopy across a striplike domain with domain size of $1 \mu \mathrm{m}$. (a) is the configuration of tip and sample magnetization assumed for calculating the magnetic dissipation and magnetic force gradient profiles. (b) and (d) are the calculated magnetic dissipation profile and force gradient profile for the $\mathrm{Co} / \mathrm{Ni}$ multilayer sample using a magnetic thin film coated Si tip. (c) and (e) are the simultaneously acquired experimental profiles of magnetic dissipation and magnetic force gradient. Data measured at constant frequency shift $\left(F^{\prime}=\right.$ constant) was converted to force gradient data $\left(F^{\prime}\right)$ by measuring the $F^{\prime}(z)$ dependence. Note the good quantitative agreement between the simple model and the experimental data. 
is for wall A [see Fig. 1(a)]. By minimizing the total energy $\gamma=\gamma_{e x}+\gamma_{a n}+\gamma_{s t a}$, we can calculate the domain wall width which oscillates with the frequency $f$. A second order approximation [in $\left(4 \mu_{0} M_{s} / K_{1} \pi\right) H_{y}$, which is much less than 1 for our experiments] of the amplitude of the domain wall width oscillation is then given by

$$
w_{1}=w_{0} \frac{2 \mu_{0} M_{s}}{K_{1} \pi} H_{y 1} \pm w_{0} \frac{12 \mu_{0}^{2} M_{s}^{2}}{K_{1}^{2} \pi^{2}} H_{y 0} H_{y 1},
$$

where $w_{0}=\sqrt{2 J S^{2} \pi^{2} / K_{1} a}$ is the wall width without external field. The difference in the width change of " $A$ " and " $B$ " domain walls comes from their different spin orientations relative to the external field. For bulk Co values of $K_{1}$ $\left(4.12 \times 10^{5} \mathrm{~J} / \mathrm{m}^{3}\right), S(1), J(1 \mathrm{eV}), a\left(2.5 \times 10^{-10} \mathrm{~m}\right)$ and $M_{s}$ $\left(1.4 \times 10^{6} \mathrm{~A} / \mathrm{m}\right)$ as well as typical tip fields of $H_{y 0}$ $=150$ Oe and $H_{y 1}=50$ Oe (see our calculations later in this letter), the second term in Eq. (1) is only $10 \%$ of the first term. The local magnetization oscillation at the domain walls leads to local elastic strain oscillation through magnetostriction in the sample, which results in energy dissipation by phonons. The energy loss in one oscillation cycle equals the elastic energy change in the sample when the wall width goes from the minimum value to the maximum value. The energy dissipation rate per unit area of the domain walls for a spatially uniform field is

$$
P=f c \lambda^{2} w_{1} .
$$

Here $c$ is the elastic constant and $\lambda$ is the magnetostriction constant of the sample. The effect of the tip field geometry will be considered later. This model predicts not only the energy dissipation but also different dissipations for different domain walls. For samples with an in-plane magnetization, Eqs. (1) and (2) are still valid for Néel walls, while for Bloch walls, $H_{y 0}, H_{y 1}$ in Eq. (1) should be replaced by the out-ofplane component of the tip field $H_{z 0}, H_{z 1}$. Depending on the tip shape, $H_{z 1}$ can be 3-5 times larger than $H_{y 1}$ according to our calculations. When considering the demagnetization energy of the wall, the anisotropy constant $K_{1}$ should be replaced by an effective anisotropy constant $K_{\text {eff }}$ whose value depends on the wall configuration and film thickness. This will lead to different dissipation as a function of magnetic wall microstructure. A difference in dissipation has indeed been observed on the cross-tie wall in a 30-nm-thick Permalloy sample. ${ }^{1}$

To test this model, we have compared experiments on various samples with numerical solutions of Eq. (2). We find good agreement between our model and experimental data on most systems studied. ${ }^{1}$ Figures 1(b)-1(e) show a comparison between simulations and experimental traces. Force gradient and dissipation data across a $1 \mu \mathrm{m}$ sized domain were simultaneously acquired on a $110-\mathrm{nm}$-thick $\mathrm{Ni} / \mathrm{Co}$ multilayer sample. A $20 \mathrm{~nm}$ sputtered CoNi coated Si tip (resonant frequency $f=80 \mathrm{kHz}$ ) at a tip-sample separation of $90 \mathrm{~nm}$ was used. The calculated minimum dissipation [Fig. 1(b)] on the domain wall positions reflects the minimum inplane field right under the tip. Figure 1(c) is the experimental damping. Note the quantitative agreement with the theoretical results. Since the film thickness is comparable with the wall width, the demagnetization energy in the wall cannot be

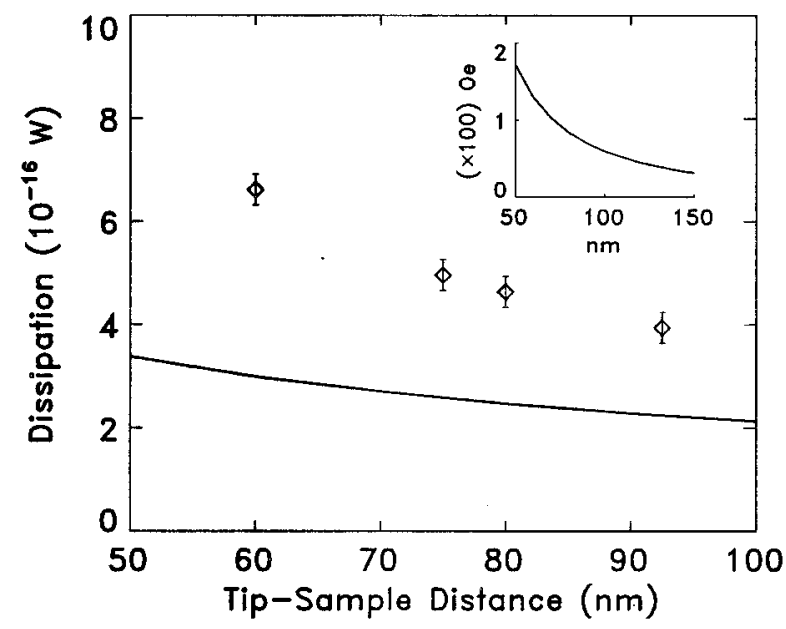

FIG. 2. Theoretical (solid line) and experimental $(\diamond)$ data for peak-peak dissipation contrast as a function of tip-sample distance using a $90 \mathrm{~nm}$ CoPtCr coated $\mathrm{Si}_{3} \mathrm{~N}_{4}$ tip on $4 \mathrm{~nm}$ Co film sample. The inset is the calculated amplitude $H_{y 1}$ of the ac part of the tip field as a function of the tip-sample distance for a peak-peak vibration amplitude of $60 \mathrm{~nm}$.

neglected. In calculating Fig. 1(b), $K_{1}$ thus needs to be replaced by $K_{\text {eff }}$, which roughly equals $K_{1}+\left(2 \mu_{0} / \pi^{2}\right) M_{s}^{2}$. Values for $K_{1}\left(2.0 \times 10^{3} \mathrm{~J} / \mathrm{m}^{3}\right), M_{s}\left(0.667 \times 10^{6} \mathrm{~A} / \mathrm{m}\right)$ and $\lambda$ $\left(-3.7 \times 10^{-5}\right)$ are from the literature. ${ }^{4}$ Other parameters $(a$, $S, J$ and $c=3.0 \times 10^{11} \mathrm{~N} / \mathrm{m}^{2}$ ) use bulk Co values. The tip field was calculated using

$$
\mathbf{H}(\mathbf{r})=\int_{\text {tip-volume }} d V^{\prime} \frac{3 \mathbf{n}\left[\mathbf{n} \cdot \mathbf{M}\left(\mathbf{r}^{\prime}\right)\right]-\mathbf{M}\left(\mathbf{r}^{\prime}\right)}{\left|\mathbf{r}-\mathbf{r}^{\prime}\right|^{3}},
$$

where $\mathbf{M}\left(r^{\prime}\right)$ is the magnetization at position $\mathbf{r}^{\prime}$ inside the tip. Based on scanning electron microscope (SEM) images, the tip was modeled to be conical in shape with a half angle of $15^{\circ}$. The tip length for the calculations was $1 \mu \mathrm{m}$ (the field for a $2 \mu \mathrm{m}$ tip was less than $10 \%$ larger than that for a $1 \mu \mathrm{m}$ tip). The bulk value of $M_{s}=667 \mathrm{emu} / \mathrm{cm}^{3}$ for the tip coating was used. The peak-peak vibration amplitude (in the $z$ direction) of the tip was $60 \mathrm{~nm}$. The calculations show that for tip-sample distances (distance from tip equilibrium point to the sample) between 250 and $50 \mathrm{~nm}$, the region of the sample for which the tip field is larger than $10 \%$ of its peak value extends over an area of less than $1.0 \mu \mathrm{m}$, with the peak field $H_{z 0}$ ranging from $10 \mathrm{Oe}$ to $160 \mathrm{Oe}$ and the ac amplitude $H_{z 1}$ of the field from 5 Oe to 80 Oe. These field values are consistent with a recent electron holography measurement on the same type of tip. ${ }^{5}$ Figure 1(d) is the calculated force gradient profile. The force gradient is calculated from $F_{z}^{\prime}$ $=\int_{\text {tip-volume }}\left(d^{2} H_{z} / d z^{2}\right) M_{s} d V^{\prime}$, where $H_{z}$ is the $z$ component of the field emanating from the sample and $M_{s}$ is the saturation magnetization of the tip thin film coating. ${ }^{6}$ Figure 1 (e) shows the force gradient profile simultaneously acquired with Fig. 1(c). The good quantitative agreement gives us confidence that the tip geometry was adequately modeled. This is important, as the tip stray field strongly influences the dissipation signal.

Figure 2 shows theoretical (solid line) and experimental (" $\diamond ")$ peak-peak damping variation as a function of the tip-sample spacings for a $4 \mathrm{~nm}$ sputtered Co film sample measured with a $90 \mathrm{~nm} \mathrm{CoPtCr}$ coated pyramidal $\mathrm{Si}_{3} \mathrm{~N}_{4}$ tip. 
In this calculation, the bulk Co values of $K_{1}, J, S, a, M_{s}, c$ and $\lambda\left(-6.0 \times 10^{-5}\right)$ were used for the sample. For such a thin Co film, the domain wall is of Néel type and the demagnetization energy in the wall can be neglected $\left(K_{\text {eff }}=K_{1}\right){ }^{3}$ With no adjustable parameters, the theory and the experiments are in quantitative agreement to within a factor of 2. The $\mathrm{Si}_{3} \mathrm{~N}_{4}$ tip was modeled to be conical in shape with a half angle of $35^{\circ}$. The cantilever's resonant frequency was 31 $\mathrm{kHz}$. Note that the dissipation increases slower than the tip field for decreasing tip-sample separation $z$. This is because magnetic dissipation depends not only on the tip field but also on the sample area over which this field extends. While the peak tip field increases with decreasing $z$, the latter decreases.

There are other possible mechanisms besides magnetoelastic effects which might lead to magnetic dissipation. We have calculated the energy dissipation due to eddy current damping both in the tip and in the sample and found that the damping is far too small to explain the experimental results. In the sample, domain wall oscillation leads to local changes in magnetic flux. The resulting eddy currents dissipate energy via ohmic heating. The effect of these currents may be quantified as a force opposed to the wall oscillation. The force is given by Ref. 7, $F=2 M v / C$ with $C=10^{9} \pi \rho /$ $(128 D M T)$. Here $v$ is the velocity of the wall edge which can be determined from Eq. (1); $M$ is saturation magnetization of the sample; $\rho$ is resistivity of the sample; $T$ is the sample thickness and $D$ is a constant close to 1 . The energy dissipation rate is given by $P_{\mathrm{dis}}=f \int_{0}^{1 / f} F v d t$, where $f$ is the resonance frequency of the cantilever. The calculated dissipation contrast for the $4 \mathrm{~nm}$ sputtered Co film with a tipsample spacing $100 \mathrm{~nm}$ is $10^{7}$ smaller than the experimental results. The bulk values of the parameters $M$ and $\rho(9.8$ $\times 10^{-6} \mu \Omega \mathrm{cm}$ ) for Co are used in the above calculations. Note that eddy current damping in the sample as a result of the flux change due to the vibrating tip does not introduce contrast in the images if the conductivity is homogeneous in the sample.

The eddy current in the tip comes from changes in the magnetic flux inside the tip when the tip is vibrated. The flux change depends on the vibration amplitude of the tip and the resonant frequency of the cantilever as well as the gradient of the field emanating from the sample. For a $\mathrm{Si}_{3} \mathrm{~N}_{4}$ tip coated with a $\mathrm{CoPtCr}$ film, the current is distributed only inside the metallic coating. The calculated tip eddy current dissipation for the $4 \mathrm{~nm}$ Co film sample is $10^{8}$ smaller than measured for a long conical tip. Even for recording tracks (10 times thicker) the signal is expected to be $10^{7}$ times smaller than the observed damping. ${ }^{1}$ This dissipation is too small to be currently detectable as the thermal limited dissipation sensi- tivity of our microscope is about $10^{-17} \mathrm{~W} .{ }^{1}$ We have experimentally tested a $\mathrm{Cu}$ coated $\mathrm{Si}_{3} \mathrm{~N}_{4}$ tip (100 nm film thickness) and have observed no damping contrast on magnetic recording tracks. Analytically, the energy dissipation due to eddy current in a rounded tip is proportional to $A^{2} f^{2} B^{\prime 2} R^{4} / \rho$. Here $A$ is the vibration amplitude of the tip, $f$ is the resonant frequency of the cantilever, $B^{\prime}$ is the gradient of the magnetic flux density generated by the sample in the tip area, $R$ is the radius of curvature at the tip apex and $\rho$ is resistivity of the tip material. By optimizing the above parameters, it might be possible to image magnetic domain structures with a nonmagnetic tip. A much smaller influence between the tip and sample can then be expected. This is an important factor, e.g., in applications of MFM to magnetic switching of ferromagnetic particles.

It should be pointed out that the local domain wall distortion due to tip field ${ }^{8}$ and tip induced rotation of spins ${ }^{9,10}$ should also contribute to the dissipation, which however are not considered in the above calculations. These mechanisms might be the origin for the faster dissipation increase in the experimental results compared to the calculated ones for decreasing tip-sample separation (see Fig. 2). We are currently in the process of attempting to quantify this effect. Note that the magnetostriction effect should be present regardless of whether other effects are contributing.

In conclusion, we have proposed a simple model based on magnetostriction which can qualitatively and semiquantitatively explain an important contribution to the energy dissipation images measured by MFM.

The authors thank James M. Freitag for providing the $\mathrm{Ni} / \mathrm{Co}$ multilayer sample. This work was supported by grants from the National Science and Engineering Research Council of Canada and Le Fonds pour la Formation des Chercheurs et l'Aide à la Recherche de la Province de Québec.

${ }^{1}$ P. Grütter, Y. Liu, P. LeBlanc, and U. Dürig, Appl. Phys. Lett. 71, 279 (1997).

${ }^{2}$ U. Dürig and H.-R. Steinauer (unpublished).

${ }^{3}$ B. D. Cullity, Introduction to Magnetic Materials (Addison-Wesley, London, 1972).

${ }^{4}$ J. Freitag, M. Sc. thesis, McGill University, 1996.

${ }^{5}$ D. G. Streblechenko, M. R. Scheinfein, M. Mankos, and K. Babcock, IEEE Trans. Magn. 32, 4124 (1996).

${ }^{6}$ D. Rugar, H. J. Mamin, P. Guethner, S. E. Lambert, J. E. Stern, I. McFadyen, and T. Yogi, J. Appl. Phys. 68, 1169 (1990).

${ }^{7}$ H. J. Williams, W. Shockley, and C. Kittle, Phys. Rev. 80, 1090 (1950).

${ }^{8}$ M. R. Scheinfein, J. Unguris, D. T. Pierce, and R. J. Celotta, J. Appl. Phys. 67, 5932 (1990).

${ }^{9}$ J. J. Sáenz, N. García, and J. C. Slonczewski, Appl. Phys. Lett. 53, 1449 (1988).

${ }^{10}$ D. W. Abraham and F. Alan McDonald, Appl. Phys. Lett. 56, 1181 (1990) 